\title{
Profile of delamanid for the treatment of multidrug-resistant tuberculosis
}

This article was published in the following Dove Press journal:

Drug Design, Development and Therapy

29 January 2015

Number of times this article has been viewed

\author{
John D Szumowski ${ }^{1,2}$ \\ John B Lynch' \\ 'Division of Allergy and Infectious \\ Diseases, Department of Medicine, \\ University of Washington, Seattle, \\ WA, USA; ${ }^{2}$ Division of AIDS Medicine, \\ Santa Clara Valley Medical Center, San \\ Jose, CA, USA
}

\begin{abstract}
New approaches to the treatment of multidrug-resistant and extensively drug-resistant tuberculosis (TB) are badly needed. Not only is the success rate of current treatment regimens suboptimal but existing regimens require multiple drugs and lengthy courses and may lead to significant toxicities. The treatment landscape is beginning to shift, however, with the recent approvals of the new TB drugs bedaquiline and delamanid. Delamanid, a dihydro-imidazooxazole, has been shown to have excellent activity against Mycobacterium tuberculosis in both in vitro and in murine TB models. It has also recently been reported to improve rates of sputum culture conversion in patients with multidrug-resistant TB when added to an optimized background regimen. Although generally well tolerated, delamanid has been associated with QT prolongation, which may be of particular clinical concern when paired with other TB drugs that may also have this effect, most notably the fluoroquinolones. Ongoing studies will help to clarify delamanid's role in the treatment of drug-resistant TB.
\end{abstract}

Keywords: delamanid, OPC-67683, Deltyba, tuberculosis

\section{Introduction}

Although shorter, better-tolerated treatment regimens would be valuable for drugsusceptible tuberculosis (TB), the need is even more acute for multidrug-resistant (MDR) and extensively drug-resistant (XDR) TB. Cases of MDR TB are resistant to the front-line drugs isoniazid and rifampicin, whereas cases of XDR TB are additionally resistant to a fluoroquinolone and at least one of the injectable anti-TB drugs such as kanamycin, amikacin, or capreomycin. ${ }^{1}$ By World Health Organization estimates, approximately 480,000 persons developed MDR TB in 2013, with 210,000 deaths from MDR TB occurring that year. ${ }^{2}$

Current treatment regimens for MDR and XDR TB are toxic, lengthy, and generally associated with low rates of clinical success. ${ }^{3}$ Encouragingly, the treatment outlook is improving, as witnessed by the recent approvals of bedaquiline and delamanid and multiple other agents under investigation. ${ }^{4,5}$ In this review, we will summarize existing data for delamanid, which was granted conditional marketing approval by the European Medicines Agency in 2014. We highlight areas where questions remain concerning delamanid's pharmacology, safety, and/or efficacy.

\section{Mechanism of action}

Delamanid ([2R]-2-methyl-6-nitro-2-[(4-\{4-[4-(trifluoromethoxy)phenoxy]-1piperidinyl phenoxy)methyl]-2,3-dihydroimidazo[2,1-b][1,3]oxazole) is a dihydroimidazooxazole previously known as OPC-67683 and is marketed under the trade name Deltyba $^{\text {TM }}$ by Otsuka Pharmaceutical Co., Ltd (Tokyo, Japan) (Figure 1). Delamanid's mechanism of action is incompletely understood but may involve inhibition of mycolic
Correspondence: John B Lynch

Harborview Medical Center, 325

9th Avenue, Box 359778, Seattle,

WA 98104, USA

Tel +l 2067445180

Fax +I 2067446564

Email jblynch@uw.edu 


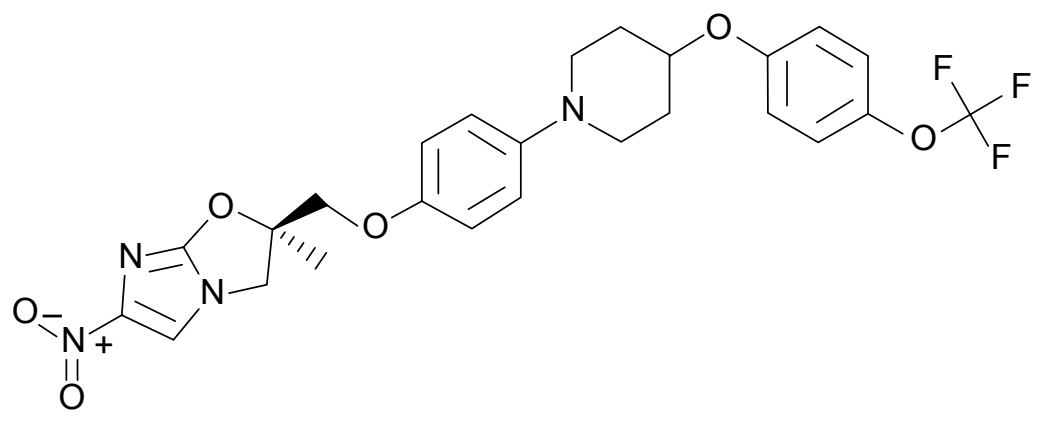

Figure I Molecular structure of delamanid.

Note: Image generated from structure data at ChemSpider. ${ }^{28}$

acid synthesis, possibly via a radical intermediate. Studies in Bacillus Calmette-Guérin showed that delamanid inhibited production of both methoxymycolic acid and ketomycolic acid. ${ }^{6}$ Like the related drug PA- $824,{ }^{7}$ delamanid has been reported to have activity against both growing and nongrowing mycobacteria. ${ }^{8}$ It is thought to be a prodrug, requiring activation by the mycobacterial F420 system, including the nitroreductase Rv3547.6,8

\section{In vitro activity}

The reported minimal inhibitory concentrations (MIC90) for delamanid range from $0.006 \mu \mathrm{g} / \mathrm{mL}$ to $0.05 \mu \mathrm{g} / \mathrm{mL}$ across Mycobacterium tuberculosis (Mtb) isolates, independent of strain resistance class (ie, pan-susceptible, MDR, or XDR strains). ${ }^{6,8,9}$ Similar results were obtained in limited testing of other members of the Mtb complex, whereas MICs were more variable for nontuberculous mycobacteria. ${ }^{8}$

Delamanid's activity was also studied in a cell culture system to model its activity against intracellular Mtb. In pulse-dose experiments, delamanid exhibited similar killing to rifampin against intracellular Mtb. ${ }^{6}$

Additional studies evaluated delamanid's ability to kill drug-tolerant TB. Such phenotypic resistance is thought to represent a major barrier to treatment shortening in TB. ${ }^{10}$ Using a liquid culture system, Saliu et $\mathrm{al}^{11}$ found that delamanid at $1 \mu \mathrm{g} / \mathrm{mL}$ showed a sterilizing effect similar to rifampin against the majority of a panel of drug-tolerant Ugandan Mtb isolates.

\section{Pharmacokinetics}

Delamanid's water solubility is poor, ${ }^{11}$ and its absorption is increased more than twofold by coadministration with food. Its absolute bioavailability is unknown but estimated to be between $25 \%$ and $47 \%$. It is more than $99 \%$ protein bound, with a high volume of distribution ( $\mathrm{Vz} / \mathrm{F}$ of 2,100 L) and a half-life of 30-38 hours. Delamanid is excreted primarily in the stool, with less than $5 \%$ excretion in the urine. It is thought to be primarily metabolized by albumin, ${ }^{8}$ with secondary contributions from P450 enzymes, primarily CYP3A4. Four major metabolites (M1-M4) have been identified in plasma in patients receiving delamanid. ${ }^{12}$ Although delamanid's metabolites have limited antimycobacterial activity, they may still contribute to QT prolongation, as discussed below. ${ }^{8,13}$

Based on toxicity in animal models, delamanid is not recommended to be used in pregnant women or women of childbearing age unless they are using contraceptives. Animal studies have also indicated that delamanid is excreted in breast milk, so it is not recommended for breastfeeding women. ${ }^{13}$

The dose of delamanid approved by the European Medicines Agency is $100 \mathrm{mg}$ twice daily. ${ }^{13}$ No dose adjustments are required for mild to moderate renal impairment, but there are no data to guide use in severe renal insufficiency. Delamanid may be used in mild hepatic impairment but is not recommended for moderate to severe hepatic impairment. A single case report describes a 12-year-old boy with XDR TB treated with delamanid, but otherwise there are no published data for delamanid's use in pediatric patients. ${ }^{14}$ There are also currently no guidelines for dose adjustment in the elderly. ${ }^{13}$

\section{Delamanid resistance}

Delamanid resistance is thought to arise from mutations in the mycobacterial F420 genes $(R v 3547, f g d, f b i A, f b i B$, $\mathrm{fbiC}$ ) associated with the prodrug's activation. In the clinical studies described here, a threshold of $0.2 \mu \mathrm{g} / \mathrm{mL}$ was used to define delamanid resistance; however, this cutoff requires further clinical validation. The spontaneous rate of delamanid resistance has been reported as $6.44 \times 10^{-6}-4.19 \times 10^{-5}$, emphasizing the need to protect delamanid with other active anti-TB drugs during therapy. ${ }^{8}$

A delamanid and PA-824 cross-resistant Mtb isolate that was obtained prior to the clinical study of either drug has been reported, ${ }^{15}$ although the molecular basis for resistance and treatment history of this isolate were not described. 
Published clinical studies have not conducted baseline delamanid susceptibility testing of Mtb isolates, although efforts are being undertaken to collect these data where possible on banked isolates. ${ }^{8}$

\section{Drug-drug interactions}

Available data suggest that delamanid does not have a significant effect on transporters such as P-glycoprotein. ${ }^{8}$ Moreover, in vitro testing suggests that delamanid neither inhibits nor induces P450 enzyme at concentrations up to $100 \mu \mathrm{M},{ }^{6,12}$ although the effects of higher concentrations (as might occur in the gut) have not been reported. ${ }^{8} \mathrm{~A}$ recent report suggests that delamanid's major metabolites also do not have inhibitory activity against hepatic P450 enzymes at concentrations achieved with standard dosing. ${ }^{12}$

In vitro checkerboard assays have shown no evidence of antagonism with the first-line anti-TB drugs isoniazid, rifampin, ethambutol, or streptomycin, ${ }^{6}$ although similar data have not been reported for drugs typically used to treat MDR or XDR TB. When given to healthy volunteers, rifampin was associated with a $47 \%$ decrease in delamanid area under the curve (AUC). ${ }^{8}$ On the other hand, ethambutol levels increased by $25 \%$ when coadministered with delamanid, though the clinical significance of this change is uncertain.

Only limited data are available concerning delamanid's interactions with antiretrovirals. Delamanid has been reported not to have a significant effect on concentrations of tenofovir, lopinavir-ritonavir, or efavirenz. ${ }^{8,16}$ Whereas lopinavir plus ritonavir was associated with a $20 \%$ increase in delamanid exposure ${ }^{16}$ and a $30 \%$ increase in delamanid's metabolite DM-6705 (see discussion in "Toxicity" following), efavirenz coadministration did not have a significant effect on delamanid levels. ${ }^{8}$ The effects of delamanid when administered with new protease inhibitors or members of other antiretroviral drug classes such as integrase inhibitors have not been reported.

\section{Murine studies}

Delamanid has shown efficacy against drug-susceptible Mtb strains in both acute and chronic murine TB models, including studies with immunodeficient mice. ${ }^{6,17}$ A delamanid-based regimen led to undetectable colony-forming units (CFU) in lung tissue at 4 months in a drug-susceptible murine model, ${ }^{6}$ suggesting that delamanid might have treatment-shortening potential. However, a relapse study was not performed, so it was unclear whether the delamanid-based regimen indeed produced more rapid sterilization as compared with the standard multidrug regimen.
Unfortunately, similar data for MDR TB have not been published. By report, however, delamanid-based regimens were associated with improved survival in mice infected with an MDR Mtb strain; further details of this study have not been described. ${ }^{8}$

\section{Human studies}

Diacon et $\mathrm{al}^{9}$ assessed the early bactericidal activity (EBA) of four doses of delamanid (100, 200, 300, and $400 \mathrm{mg}$ once daily) in human immunodeficiency virus (HIV)-negative persons with rifampin-susceptible TB. The average EBA for the delamanid groups was modest $\left(0.04 \log _{10} \mathrm{CFU} / \mathrm{mL}\right.$ sputum/day). No statistically significant differences between the groups were observed, with the caveat that the groups were small and there was significant intragroup variability in delamanid levels. In contrast, there did appear to be a relationship between EBA and delamanid levels; later unpublished work suggested that maximal EBA correlated with an AUC between 3,500 $\mathrm{ng}{ }^{*} \mathrm{hr} / \mathrm{mL}$ and 5,500 $\mathrm{ng} * \mathrm{hr} / \mathrm{mL}$. ${ }^{18}$ In the pharmacokinetic component of Diacon et al's ${ }^{9}$ study, the investigators observed that delamanid exposure did not increase proportionate to dose and plateaued with $300 \mathrm{mg}$ daily dosing.

In a subsequent, Phase II trial (Trial 204), 481 participants with MDR TB were treated with delamanid $100 \mathrm{mg}$ twice daily, delamanid $200 \mathrm{mg}$ twice daily, or placebo combined with an optimized background regimen (OBR) for 8 weeks and then followed for an additional 4 weeks with the OBR alone. The primary end point of this study was culture conversion on liquid media (mycobacteria growth indicator tube) at 8 weeks. Of note, the study excluded persons with baseline QT prolongation or conduction delay on electrocardiogram (ECG), as well as persons being treated with moxifloxacin. Few HIV-positive persons were enrolled $(n=4)$; none was on antiretrovirals and all had CD4 counts of at least 350 cells $/ \mu \mathrm{L}$. The investigators found that both doses of delamanid were associated with increased rates of mycobacteria growth indicator tube culture negativity at 8 weeks (45.4\% for delamanid $100 \mathrm{mg}$ twice daily and $41.9 \%$ for delamanid $200 \mathrm{mg}$ twice daily vs $29.6 \%$ for OBR alone); similar results were seen with solid media. In general, delamanid was well tolerated. Significantly higher proportions of participants receiving delamanid experienced QT prolongation, and the frequency appeared to be dose dependent $(9.9 \%$ for delamanid $100 \mathrm{mg}$ twice daily and $13.1 \%$ for delamanid $200 \mathrm{mg}$ twice daily vs 3.8\% for OBR alone); none of these cases was associated with clinical manifestations such as arrhythmia or syncope. ${ }^{19,20}$ 
Although culture conversion at 8 weeks (traditionally assessed on solid media) is a commonly accepted surrogate end point in trials for drug-susceptible $\mathrm{TB},{ }^{21}$ its clinical significance for MDR TB is less established. However, observational studies have reported higher rates of good clinical outcomes (cure or completion of treatment) in MDR patients achieving culture conversion around 2 months, ${ }^{22,23}$ suggesting that the higher rates of culture conversion at 8 weeks with delamanid-based regimens observed in Trial 204 may indeed be clinically meaningful.

In order to further assess the safety and efficacy of longer durations of delamanid treatment that would better represent its use in clinical practice, an open-label extension to Trial 204 was undertaken (Trial 208). In this investigation, participants were treated with an additional 6 months of delamanid at $100 \mathrm{mg}$ twice daily (later increasing to $200 \mathrm{mg}$ twice daily at the investigator's discretion) plus the OBR, starting at a minimum of 4 weeks after completing delamanid or placebo in Trial 204. There are two additional, important caveats to Trial 208, however. First, less than half (213/481, 44\%) of Trial 204 participants had the option to participate in Trial 208 or chose to participate in Trial 208. Second, there was a significant delay in starting Trial 208 at many sites; at more than a third, the delay in starting delamanid was more than 4 months. ${ }^{8,18}$

Independent of their participation in the delamanid open-label extension study (Trial 208), participants in Trial 204 could be enrolled into an observational cohort for longitudinal follow-up (Study 116). Approximately 88\% (421/481) of participants in Trial 204 chose to do so. In Study 116, participants were followed for up to 24 months following their first dose of delamanid in Trial 204 or until the end of MDR treatment, whichever came first. Treatment and monitoring in Study 116 were done according to local standard practice. ${ }^{18}$

Among Study 116 participants, a significantly higher proportion of persons who received delamanid at any dose for 6-8 months (ie, those who had participated in Trial 208) achieved a favorable outcome of cure or treatment completion compared with participants who received 0-2 months of delamanid ( $74.5 \%$ vs $55.0 \%$ ). Mortality was also reduced among those receiving $6-8$ months of delamanid ( $1 \%$ vs $8.3 \%) .{ }^{18}$ Although these results are promising, serious limitations in the study design limit the conclusions that can be drawn from Trial 208 and Study 116. Most significant is the potential for selection bias. Participants in Trial 204 who did not elect to participate or have the opportunity to participate in Trial 208 may have been sicker or less adherent than those who did choose to participate, which could account for lower rates of good clinical outcomes among persons receiving only $0-2$ months of delamanid. Indeed, 8 -week culture conversion rates were higher among Trial 204 participants who subsequently continued in Trial 208, compared with those who did not. ${ }^{8}$ Unfortunately, no other data are published comparing characteristics of persons who participated or did not participate in Trial 208.

\section{Toxicity}

Given the general poor tolerability of the drugs commonly used to treat MDR and XDR TB, it can be challenging to assign causality for reported adverse effects to new TB drugs. In clinical studies of delamanid, the most frequent adverse drug reactions include nausea (38\%), vomiting (33\%), and dizziness $(30 \%)$. Other notable adverse reactions included tremor, paresthesia, and anxiety. ${ }^{13}$

While symptomatic management may mitigate these reactions, delamanid's potential to cause QT prolongation is more concerning. Indeed, patients undergoing treatment for MDR and XDR TB often have multiple other risk factors for QT prolongation, ${ }^{24}$ such as electrolyte disturbances or treatment with other concomitant medications such as clofazimine, bedaquiline, or fluoroquinolones that have been associated with QT prolongation. As noted previously, approximately $10 \%$ of participants receiving delamanid in Trial 204 experienced QT prolongation, but only one individual developed QT prolongation over $500 \mathrm{~ms}$. There was a trend toward more palpitations in the delamanid group, but more serious cardiac events were not observed. Of note, cases with QT prolongation over $60 \mathrm{~ms}$ were associated with concomitant fluoroquinolone treatment (either levofloxacin or ofloxacin, as moxifloxacin use was not permitted in Trial 204). Further analysis showed that risk of QT prolongation among persons receiving delamanid was strongly associated with hypoalbuminemia; albumin under $2.8 \mathrm{~g} / \mathrm{dL}$ is therefore listed as a contraindication to delamanid treatment. ${ }^{13}$

Delamanid's effects on the QT interval appear to be dose related and increase over the initial 6-10 weeks of treatment. The effect has been linked to delamanid's major metabolite DM-6705. Both CYP3A4 inducers and inhibitors may increase levels of DM-6705, necessitating more intensive cardiac monitoring in such settings. ${ }^{8,13}$ Current recommendations call for, at a minimum, a baseline and monthly ECG for persons on delamanid; delamanid is not recommended to be started or continued if the corrected QT interval is over $500 \mathrm{~ms}^{13}$ 


\section{Ongoing and planned clinical studies}

Planned or ongoing clinical studies will help address gaps in current knowledge relating to delamanid's use in MDR or XDR TB. A pediatric Phase I study will assess safety and pharmacokinetics of delamanid given for 10 days to 6- to 18-year-olds with MDR TB receiving an OBR (Otsuka 24212-232, clinicaltrials.gov NCT01856634). Children aged 12-17 years will receive delamanid $100 \mathrm{mg}$ twice daily, whereas children aged 6-11 years will receive $50 \mathrm{mg}$ twice daily. A 6-month open-label study of delamanid plus OBR will follow the Phase I investigation, and younger age groups will also be included once the appropriate drug formulations are available.

A multisite Phase III clinical trial (Otsuka 242-09-213, clinicaltrials.gov NCT01424670) will evaluate a 6-month course of delamanid versus placebo plus OBR among adults with MDR TB. In contrast to earlier studies, delamanid dosing will be $100 \mathrm{mg}$ twice daily for 2 months followed by $200 \mathrm{mg}$ once daily for 4 months. A substudy of HIV-positive participants receiving antiretroviral therapy is planned as part of this trial.

\section{Remaining questions and conclusion}

Delamanid is likely to provide a valuable addition to current therapies for MDR and XDR TB, yet multiple questions remain concerning its clinical use. First, the optimum duration of delamanid therapy is uncertain; limited quality data suggest that the duration should be at least 6 months, ${ }^{18}$ but whether there would be additional benefit to longer treatment has not been established. It is also unclear whether the dose and schedule of delamanid approved by the European Medicines Agency, $100 \mathrm{mg}$ twice daily, is the most appropriate; further studies are planned to assess this question. ${ }^{8,25}$ Furthermore, delamanid's ability to protect against emergence of resistance to other coadministered anti-TB drugs and its interactions with newer antiretroviral regimens remain to be studied. As is the case for most other TB drugs, it is unclear which agents would be the most effective and least toxic when paired with delamanid to treat MDR or XDR TB. ${ }^{26}$

Given delamanid's potential to prolong the QT interval, monitoring for cardiac toxicities will be essential as the drug is studied in larger patient populations. How and whether it can be safely used with other anti-TB drugs that have QTprolonging potential, such as bedaquiline, clofazimine, and newer-generation fluoroquinolones such as moxifloxacin is an important unanswered practical question for TB clinicians. These cardiac concerns will restrict delamanid's use to health centers that are comfortable performing and interpreting ECGs.

The failure of fluoroquinolone-based regimens to permit treatment shortening for drug-susceptible TB has raised concerns about the predictive value of sputum culture conversion rates for relapse-free TB cure in the modern era. ${ }^{27}$ It will therefore be important to more rigorously determine whether delamanid-based regimens indeed translate into improved clinical outcomes and lower overall mortality in patients with MDR or XDR TB.

Despite these outstanding questions, the medical need for improved MDR and XDR therapies is great, and the published data for delamanid are promising. These considerations led to the conditional marketing approval of delamanid by the European Medicines Agency in 2014. TB clinicians and patients await further results from the ongoing clinical studies of delamanid with great interest.

\section{Disclosure}

The authors report no conflicts of interest in this work.

\section{References}

1. Zumla A, Raviglione M, Hafner R, von Reyn CF. Tuberculosis. NEngl J Med. 2013;368(8):745-755.

2. Multidrug-resistant tuberculosis (MDR-TB) 2014 Update. World Health Organization; 2014.

3. Ahuja SD, Ashkin D, Avendano M, et al. Multidrug resistant pulmonary tuberculosis treatment regimens and patient outcomes: an individual patient data meta-analysis of 9,153 patients. PLoS Med. 2012;9(8):e1001300.

4. Zumla AI, Gillespie SH, Hoelscher M, et al. New antituberculosis drugs, regimens, and adjunct therapies: needs, advances, and future prospects. Lancet Infect Dis. 2014;14(4):327-340.

5. Dooley KE, Nuermberger EL, Diacon AH. Pipeline of drugs for related diseases: tuberculosis. Curr Opin HIV AIDS. 2013;8(6):579-585.

6. Matsumoto M, Hashizume H, Tomishige T, et al. OPC-67683, a nitrodihydro-imidazooxazole derivative with promising action against tuberculosis in vitro and in mice. PLoS Med. 2006;3(11):e466.

7. Stover CK, Warrener P, VanDevanter DR, et al. A small-molecule nitroimidazopyran drug candidate for the treatment of tuberculosis. Nature. 2000;405(6789):962-966.

8. Assessment report Deltyba, Procedure No.: EMEA/H/C/002552. European Medicines Agency, Committee for Medicinal Products for Human Use; 2013.

9. Diacon AH, Dawson R, Hanekom M, et al. Early bactericidal activity of delamanid (OPC-67683) in smear-positive pulmonary tuberculosis patients. Int J Tuberc Lung Dis. 2011;15(7):949-954.

10. Connolly LE, Edelstein PH, Ramakrishnan L. Why is long-term therapy required to cure tuberculosis? PLoS Med. 2007;4(3):e120.

11. Saliu OY, Crismale C, Schwander SK, Wallis RS. Bactericidal activity of OPC-67683 against drug-tolerant Mycobacterium tuberculosis. J Antimicrob Chemother. 2007;60(5):994-998.

12. Shimokawa Y, Sasahara K, Yoda N, Mizuno K, Umehara K. Delamanid does not inhibit or induce cytochrome p450 enzymes in vitro. Biol Pharm Bull. 2014;37(11):1727-1735.

13. Deltyba EPAR Product Information, Annex I summary of product characteristics. European Medicines Agency, Committee for Medicinal Products for Human Use; 2014. 
14. Esposito S, D'Ambrosio L, Tadolini M, et al. ERS/WHO Tuberculosis Consilium assistance with extensively drug-resistant tuberculosis management in a child: case study of compassionate delamanid use. Eur Respir J. 2014;44(3):811-815.

15. Doi N, Disratthakit A. Characteristic Anti-Mycobacterial Spectra of the Novel Anti-TB Drug Candidates OPC-67683 and PA-824. Interscience Conference on Antimicrobial Agents and Chemotherapy 2006; San Francisco, CA.

16. Paccaly AJ, Petersen C, Patil S, et al. Absence of clinically relevant drug interaction between delamanid, a new drug for multidrug-resistant tuberculosis (MDR-TB) and tenofovir or lopinavir/ritonavir in healthy subjects. 19th International AIDS Conference; 2012.

17. Sasaki H, Haraguchi Y, Itotani M, et al. Synthesis and antituberculosis activity of a novel series of optically active 6-nitro-2, 3-dihydroimidazo[2,1-b]oxazoles. J Med Chem. 2006;49(26): 7854-7860.

18. Skripconoka V, Danilovits M, Pehme L, et al. Delamanid improves outcomes and reduces mortality in multidrug-resistant tuberculosis. Eur Respir J. 2013;41(6):1393-1400.

19. Gler MT, Skripconoka V, Sanchez-Garavito E, et al. Delamanid for multidrug-resistant pulmonary tuberculosis. $N$ Engl J Med. 2012;366(23):2151-2160.

20. Zhang Q, Liu Y, Tang S, Sha W, Xiao H. Clinical benefit of delamanid (OPC-67683) in the treatment of multidrug-resistant tuberculosis patients in China. Cell Biochem Biophys. 2013;67(3):957-963.
21. Mitchison DA. Assessment of new sterilizing drugs for treating pulmonary tuberculosis by culture at 2 months. Am Rev Respir Dis. 1993;147(4):1062-1063.

22. Holtz TH, Sternberg M, Kammerer S, et al. Time to sputum culture conversion in multidrug-resistant tuberculosis: predictors and relationship to treatment outcome. Ann Intern Med. 2006;144(9):650-659.

23. Rodriguez M, Monedero I, Caminero JA, et al. Successful management of multidrug-resistant tuberculosis under programme conditions in the Dominican Republic. Int J Tuberc Lung Dis. 2013;17(4):520-525.

24. Owens RC Jr, Nolin TD. Antimicrobial-associated QT interval prolongation: pointes of interest. Clin Infect Dis. 2006;43(12): 1603-1611.

25. Diacon AH, von Groote-Bidlingmaier F, Donald PR. Delamanid, a new 6-nitro-2,3-dihydroimidazo[2,1-b] oxazole for the management of tuberculosis resistant to at least isoniazid and rifampicin. Expert Opin Orphan Drugs. 2014;2(1):87-94.

26. Olaru ID, von Groote-Bidlingmaier F, Heyckendorf J, Yew WW, Lange C, Chang KC. Novel drugs against tuberculosis: a clinician's perspective. Eur Respir J. Epub 2014 Nov 27.

27. Warner DF, Mizrahi V. Shortening treatment for tuberculosis: to basics. N Engl J Med. 2014;371(17):1642-1643.

28. Delamanid [webpage on the internet]. Available from: http://www. chemspider.com/Chemical-Structure.4981055.html. Accessed January 8, 2015 .
Drug Design, Development and Therapy

\section{Publish your work in this journal}

Drug Design, Development and Therapy is an international, peerreviewed open-access journal that spans the spectrum of drug design and development through to clinical applications. Clinical outcomes, patient safety, and programs for the development and effective, safe, and sustained use of medicines are a feature of the journal, which

\section{Dovepress}

has also been accepted for indexing on PubMed Central. The manuscript management system is completely online and includes a very quick and fair peer-review system, which is all easy to use. Visit http://www.dovepress.com/testimonials.php to read real quotes from published authors.

Submit your manuscript here: http://www.dovepress.com/drug-design-development-and-therapy-journal 